\title{
Characterization of bidirectional impulse turbines for thermoacoustic engines
}

Michael A. G. Timmer, and Theo H. van der Meer

Citation: The Journal of the Acoustical Society of America 146, 3524 (2019); doi: 10.1121/1.5134450

View online: https://doi.org/10.1121/1.5134450

View Table of Contents: https://asa.scitation.org/toc/jas/146/5

Published by the Acoustical Society of America

\section{ARTICLES YOU MAY BE INTERESTED IN}

Review on the conversion of thermoacoustic power into electricity

The Journal of the Acoustical Society of America 143, 841 (2018); https://doi.org/10.1121/1.5023395

Evaluations of acoustic damping performances of double-layer in-duct perforated plates at low Mach and Helmholtz number

The Journal of the Acoustical Society of America 146, 3512 (2019); https://doi.org/10.1121/1.5134063

Optimizing bidirectional impulse turbines for thermoacoustic engines

The Journal of the Acoustical Society of America 147, 2348 (2020); https://doi.org/10.1121/10.0001067

Effect of surface roughness on nonlinear reflection of weak shock waves

The Journal of the Acoustical Society of America 146, EL438 (2019); https://doi.org/10.1121/1.5133737

Underwater radiated noise from hydrofoils in coastal water

The Journal of the Acoustical Society of America 146, 3552 (2019); https://doi.org/10.1121/1.5134779

Accuracy of Green's function estimation from correlation of diffuse elastic waves on thin plates

The Journal of the Acoustical Society of America 146, 3505 (2019); https://doi.org/10.1121/1.5134066

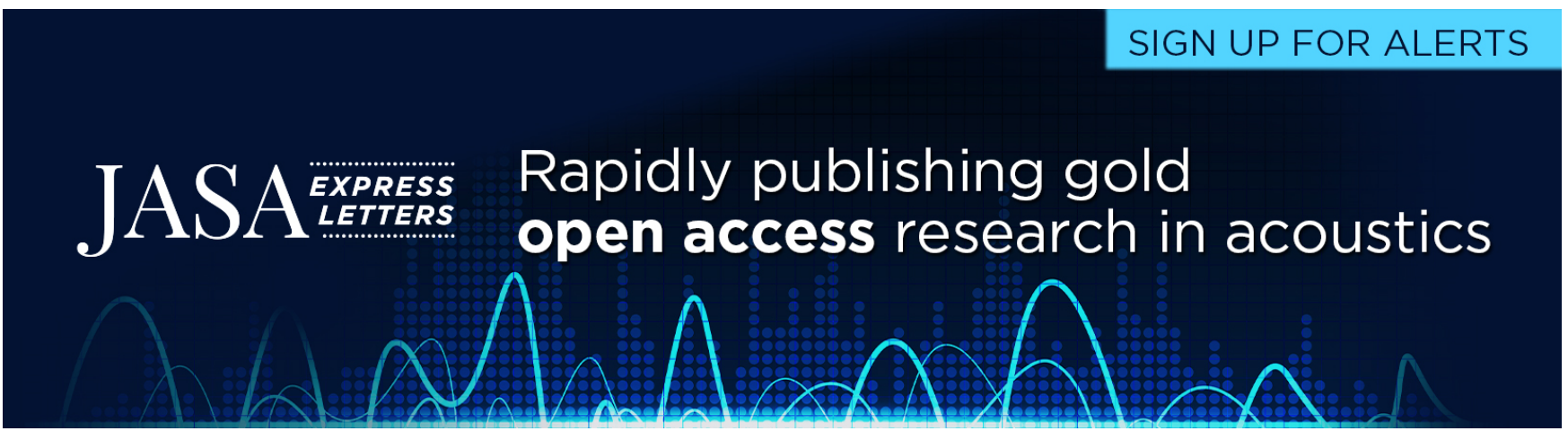




\title{
Characterization of bidirectional impulse turbines for thermoacoustic engines
}

\author{
Michael A. G. Timmer ${ }^{\mathrm{a})}$ and Theo H. van der Meer \\ Department of Thermal Engineering, University of Twente, Enschede, The Netherlands
}

(Received 22 August 2019; accepted 28 October 2019; published online 25 November 2019)

\begin{abstract}
A bidirectional impulse turbine to convert thermoacoustic power into electricity is investigated. Experimental measurements are done with a loudspeaker for varying acoustic conditions and turbine loads. The results are used to characterize the turbine performance and compare it to steady flow turbomachinery and turbines in oscillating water columns. A dimensional analysis is done to identify the variables that influence the turbine performance, after which a scaling is determined that uniquely determines the efficiency of the turbine. The work is finished by providing the impedance of the bidirectional turbine such that it can be implemented in a thermoacoustic engine.
\end{abstract}

(c) 2019 Acoustical Society of America. https://doi.org/10.1121/1.5134450

[JDM]

Pages: $3524-3535$

\section{INTRODUCTION}

The conversion of heat into acoustic power is known as the thermoacoustic effect. Devices can utilize this effect by extracting energy from a heat source and converting it into useful power. Such thermoacoustic devices can start working for a temperature gradient with the environment as small as $30 \mathrm{~K},{ }^{1}$ which is why the main application is usually seen in harvesting low-grade heat from, e.g., solar heat ${ }^{2,3}$ or waste heat. ${ }^{4}$ The focus of thermoacoustic devices is mainly on refrigeration, but the power in the acoustic wave can also be converted into mechanical work and electricity. A promising use case of this power is to drive the pumps and ambient circuit of a thermoacoustic refrigerator, therewith providing completely off grid cooling from only a heat source.

Usually, acoustic power is converted into electricity by the use of linear alternators, which can be regarded as dedicated loudspeakers that are driven in reverse. A literature review on the conversion of acoustic power into electricity has shown that bidirectional turbines are an attractive alternative to the linear alternators, especially when scaling up to industrial sizes. ${ }^{5}$ The origin of bidirectional turbines lies in the field of oscillating water columns (OWCs), where they are used to harvest the energy from ocean waves near shorelines. ${ }^{6,7}$ The special design of these turbines ensures that the rotation of the rotor is independent of the axial flow direction, making them well suited to also convert acoustic waves into power.

In 2014, it was shown that a bidirectional impulse turbine does indeed work under thermoacoustic conditions. ${ }^{8}$ In another work, several Wells and impulse turbines have been tested in the scope of thermoacoustics, yet without a load attached to the turbine and only for unidirectional flow. ${ }^{9}$ The underlying assumption for such unidirectional tests is that the oscillating flow in question can be assumed to be quasisteady, which is indeed shown to be the case for OWCs. ${ }^{10,11}$ Moisel and Carolus present a derivation to show that the effect of the inertia of the accelerated or decelerated fluid in

${ }^{\text {a)} E l e c t r o n i c ~ m a i l: ~ t i m m e r . m a g @ g m a i l . c o m ~}$ the turbine can be neglected in OWCs. ${ }^{11}$ They conclude this for ocean waves with a frequency of around $0.1 \mathrm{~Hz}$, with the note that this inertial effect becomes more relevant for higher frequencies such as $1 \mathrm{~Hz}$. Since thermoacoustic engines generally work in the range of 20 to $200 \mathrm{~Hz}$, it is questionable whether the flow in the bidirectional turbines can still be regarded as quasi-steady under these circumstances. Since most of the performance characteristics used for turbines in OWCs are also based on steady flow, it is unclear whether the results from this field are still applicable for thermoacoustics. Besides this, it is quite unknown in general how well a bidirectional turbine performs under varying thermoacoustic conditions. These topics have remained open since the literature on bidirectional turbines in thermoacoustics is very scarce. The main aim of this work is to alleviate some of this uncertainty by characterizing the performance of a bidirectional impulse turbine under varying thermoacoustic conditions.

The experimental setup that is used for this work is presented in Sec. II, along with the turbine design and the measurement procedure. Special care is taken for calibrating and validating the experimental setup, in an effort to build a foundation for bidirectional turbine measurements in thermoacoustics. In Sec. III, the turbine performance under varying thermoacoustic conditions is presented and characterized, concluding with a part that focuses on the turbine impedance and how to implement it in a thermoacoustic engine.

\section{EXPERIMENTAL SETUP}

The experimental set-up is shown schematically in Fig. 1. This set-up, previously used by Aben ${ }^{12}$ and Oosterhuis, ${ }^{13}$ has been adjusted for the current work to incorporate the electrical load to the turbine's generator and the measurement thereof. On the right hand side, a 15 in. loudspeaker (JBL W15GTi) is enclosed in a cylindrical back volume. This section is structurally decoupled from the rest of the setup by a membrane. In its current state, the system cannot be pressurized; thus, all measurements are done with air at ambient pressure. A $2 \mathrm{~kW}$ 


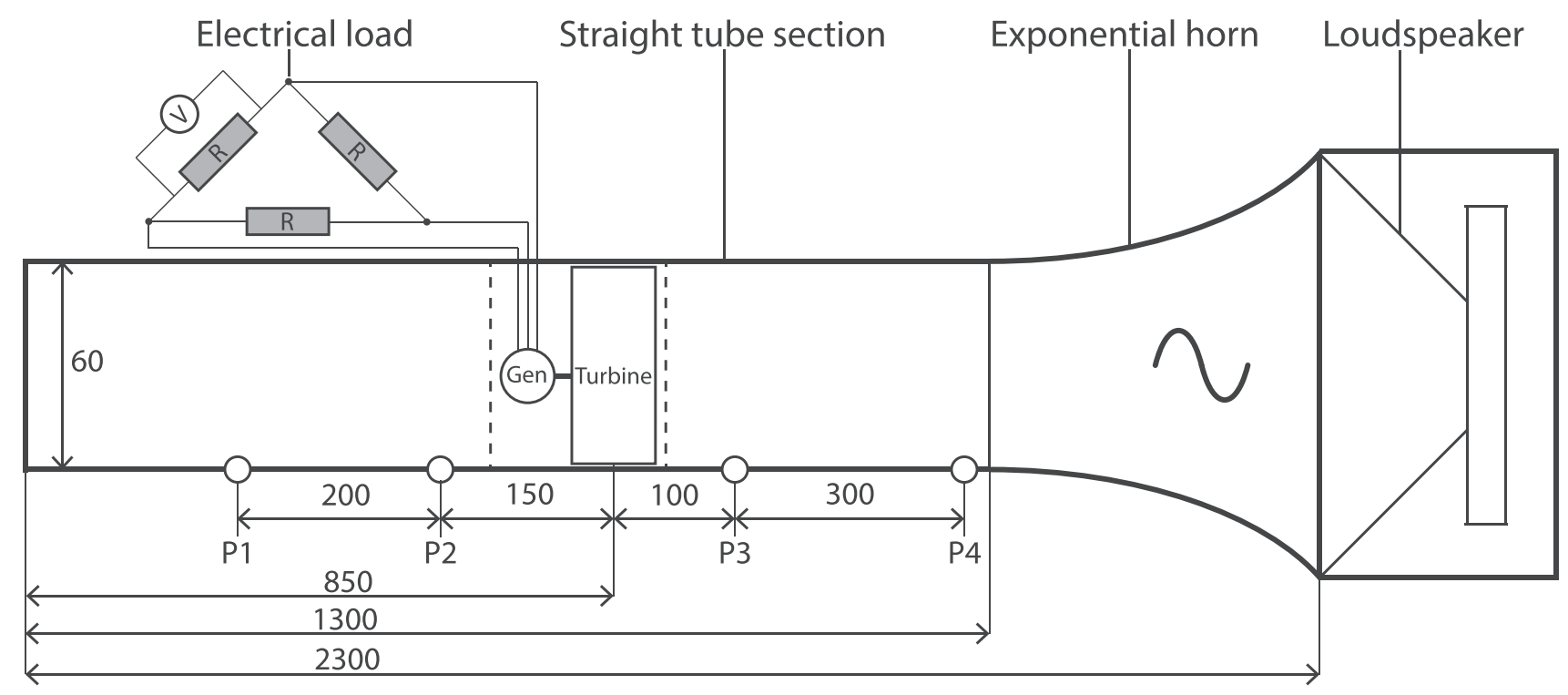

FIG. 1. Schematic of the experimental setup (not to scale). The pressure sensor locations (P1-P4) and tube dimensions are given in mm. A detailed schematic of the turbine section (depicted by the dashed lines) is given in Fig. 2.

audio amplifier (Behringer EP2000) drives the loudspeaker with an audio signal from a computer sound card. The acoustic wave travels through an exponentially contracting horn to the straight tube section where turbines can be mounted and tested. To acquire the largest acoustic amplitudes with the given setup, a closed end is used on the left, resulting in pressure amplitudes of up to $7500 \mathrm{~Pa}$. The latter is measured by four piezoresistive differential pressure sensors (Honeywell 26PCAFA6D) mounted flush with the tube wall. As shown by P1-P4 in Fig. 1, there are two sensors on either side of the turbine to be able to calculate the local acoustic power. More details on these calculations is given in Sec. II B and the calibration of the sensors is presented in Sec. II C.

The $60 \mathrm{~mm}$ diameter test section is modular in length, but kept at $1300 \mathrm{~mm}$ in this work, such that there is sufficient acoustic power for frequencies in the range $50-90 \mathrm{~Hz}$. To perform measurements for substantially lower or higher acoustic frequencies, the test section has to be lengthened or shortened, respectively. The turbine and generator combination is mounted in a $100 \mathrm{~mm}$ tube section (shown by the dashed lines in Fig. 1). The mid-plane of the turbine is at $850 \mathrm{~mm}$ from the closed end, where the acoustic velocity amplitude is large enough for significant turbine performance for all measurement frequencies. A Hacker A10-13L brushless electric motor is connected to the rotor and used as a generator. The induced three-phase alternating current of the generator is connected to three $20 \mathrm{~W}$ precision resistors in delta configuration, as denoted by the electrical load in Fig. 1. Different sets of $\mathrm{R}=4.7,10$, and $20 \mathrm{Ohm}$ resistors are used to be able to have a variable rotational velocity of the turbine for the same acoustic input. The calibration of the generator efficiency for all electrical loads is described in

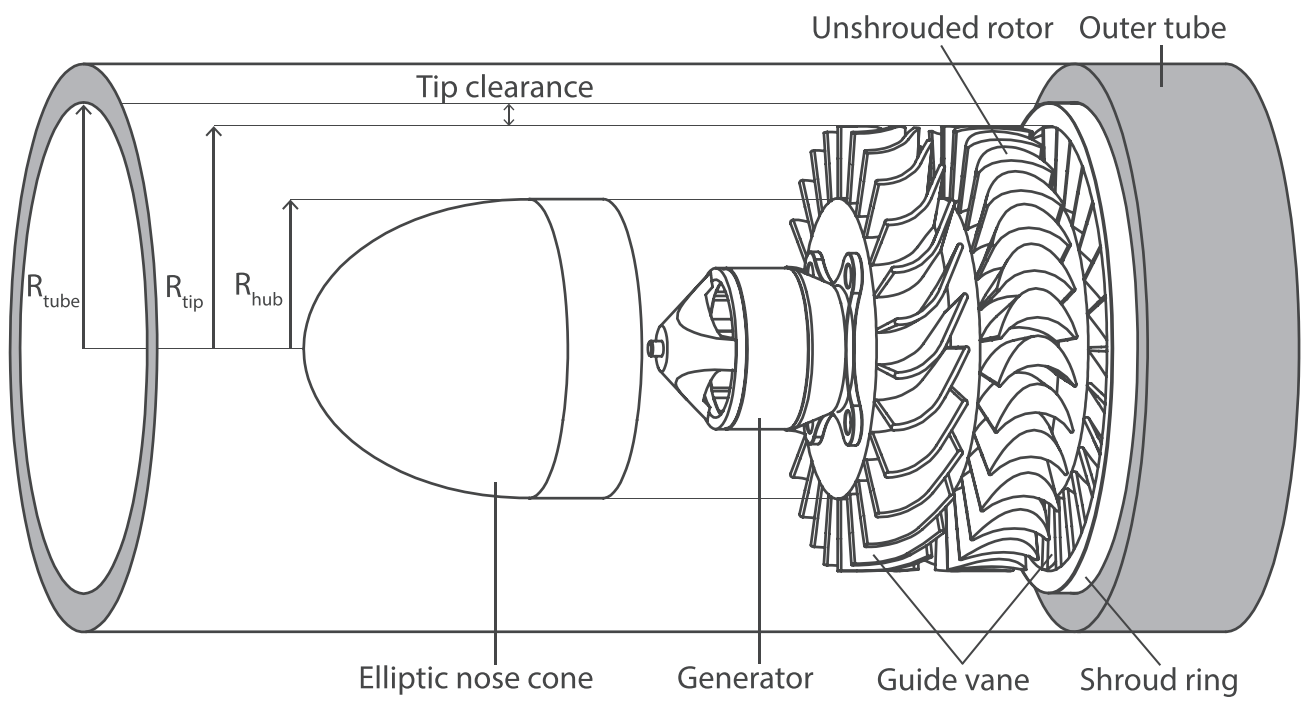

FIG. 2. Schematic of an axial bidirectional impulse turbine mounted in a tube section. The guide vanes are connected to the outer tube by a shroud ring (only depicted for the right guide vane). The generator is mounted on the left guide vane and connected to the rotor. During operation, the generator is enclosed by the nose cone, which also ensures the axial flow is redirected towards the guide vane blades. 
Sec. II C. Note that extra clamps have been placed on the generator axis to prevent axial movement of the rotor and axis. High speed imaging has shown that after placing these clamps no significant axial movement is left. The latter is especially important for measurements where the spacing between the guide vanes and rotor is small, such as the $1 \mathrm{~mm}$ spacing used in this work.

During each experiment, the four pressure signals, the power to the loudspeaker, and the voltage over one resistor of the electrical load are measured with an NI-6250 data acquisition system. This is done by sampling at $20 \mathrm{kHz}$ for a duration of twenty seconds per loudspeaker setpoint, of which the first ten seconds are discarded to allow the turbine to reach a constant operating speed. The remaining raw data are digitally phase-locked and processed, after which the results are stored in the TDMS file format for further processing. The TDMS files, further processing scripts, and final results can be found in a data publication ${ }^{14}$ for the measurements presented in this work.

\section{A. Turbine design}

For the conversion of acoustic power into electricity, an axial impulse turbine is used that is based on the work from Suzuki et al. for an OWC. ${ }^{15}$ A schematic of this turbine mounted in a tube section is shown in Fig. 2. This turbine can convert acoustic power into shaft power by redirecting the bidirectional axial flow towards the rotor blades with two sets of guide vanes. At the rotor blades, the flow induces a torque, causing a rotation in the direction given in Fig. 3.
The resulting shaft power is converted into electricity by connecting a generator to the rotor.

The turbine has 29 rotor blades with 26 guide vanes on either side of it. Instead of the 30 blades by Suzuki et al., ${ }^{15}$ it is chosen to use a prime number of rotor blades to avoid any planes of symmetry. The guide vanes are connected to the outer tube by a shroud ring (only depicted for the right guide vane). The rotor in this work does not have a shroud ring, but this could be implemented in an effort to reduce radial leakage. The tip clearance is the radial spacing between the tip of the blade and the outer tube, as shown in Fig. 2. This is set to $1 \mathrm{~mm}$ in this work, resulting in a hub-to-tip ratio of 0.68 .

To guide the axial flow towards the blades, nose cones are connected to both guide vanes. The cones have an axial length of $40 \mathrm{~mm}$, consisting of a $30 \mathrm{~mm}$ elliptic part running up to the hub radius, followed by a $10 \mathrm{~mm}$ straight part. Figure 2 depicts the nose cone in an exploded view to show the generator that is connected to the left guide vane. In practice, the nose cone is connected to the guide vane and covers the generator, leaving only room for the electrical wires to pass towards the outer tube and electrical load. The turbine and nose cones are 3D printed from PA 2200 plastic using selective laser sintering. The accuracy and influence of the $3 \mathrm{D}$ printing technique on the turbine performance is presented in Sec. II D.

The exact shape of the guide vanes and rotor blades is shown to scale in Fig. 3. The design is presented in such a way that the given dimensions and relations exactly determine the geometry and a design study for the length, thickness and angle of the rotor blade can easily be carried out (in

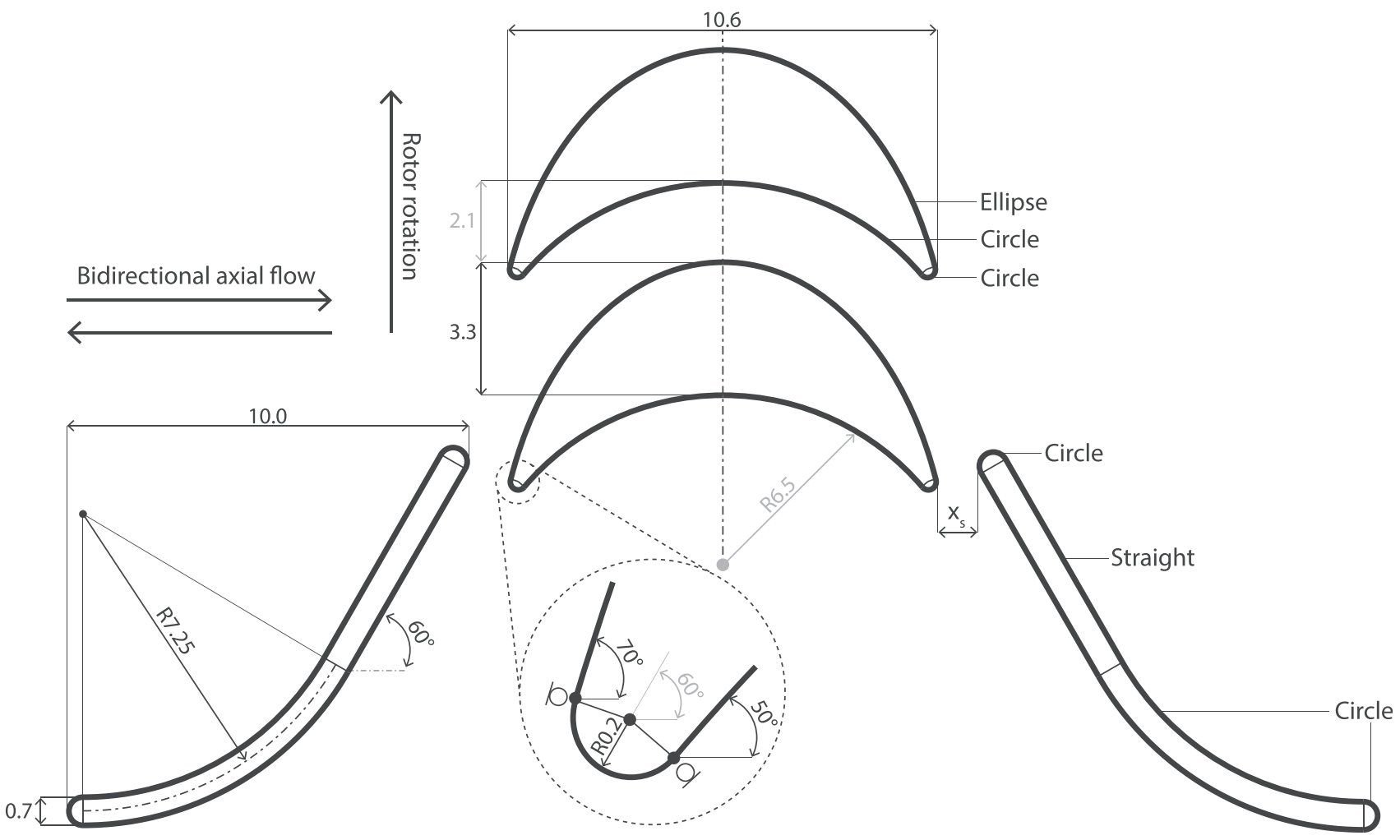

FIG. 3. Schematic of the bidirectional impulse turbine (to scale). The dimensions are given in mm unless stated otherwise, and provided at the mean turbine radius of $24.8 \mathrm{~mm}$. The dimensions in black are exact (not rounded) and completely determine the geometry. The values in gray follow from the defined geometry and turbine properties, such as number of blades. Note that in the zoomed section the symbol refers to lines being tangent at the corresponding points ( ) at the given angles. These $50^{\circ}$ and $70^{\circ}$ angles ensure an effective angle of $60^{\circ}$ between the centerline of the rotor blade and the axial flow direction. 
potential future work). With a fixed hub radius of $19.6 \mathrm{~mm}$ and tube radius of $30 \mathrm{~mm}$, the given geometry from literature $^{15}$ was scaled to the mean radius of $24.8 \mathrm{~mm}$. The axial spacing between the guide vanes and rotor blades, depicted by $\mathrm{X}_{\mathrm{s}}$ in Fig. 3 , is set to $1 \mathrm{~mm}$.

\section{B. Performance calculations}

To measure the input power absorbed by the turbine, the acoustic power before and after the turbine has to be known. Acoustic power is defined as

$$
E_{2}=\frac{1}{2}\left|p_{1}\right|\left|U_{1}\right| \cos \left(\phi_{p U}\right)
$$

with $p_{1}$ the complex pressure amplitude, $U_{1}$ the complex flowrate, and $\phi_{p U}$ the phase angle between $p_{1}$ and $U_{1}$. Since acoustic power is a difficult property to directly measure, it is approximated with sets of two pressure sensors using the following procedure. The atmospheric pressure and ambient temperature are measured and used to calculate the current gas properties of the air. The complex amplitudes of the pressure signals are determined by performing a discrete Fourier transform at the driving frequency and adding the phase information. The complex pressure amplitudes are subsequently used to determine the complex velocity amplitude and the acoustic power using Eqs. (12) and (23) of Fusco et al., ${ }^{16}$ respectively. These calculations include the effect of viscosity for accurately measuring at the tube wall and are valid for an arbitrary distance. Note that the calculated values are located in the middle of both pressure sensor sets P1-P2 (left of the turbine) and P3-P4 (right of the turbine). This is extended to the complete complex pressure and velocity fields on both sides of the turbine by solving Eqs. (4.54) and (4.70) from Swift with the boundary layer approximation. ${ }^{17}$ The acoustic power dissipation in the tube between the turbine and sensor location is then corrected for using Eq. (5.11) of Swift, ${ }^{17}$ resulting in the acoustic power absorbed solely by the turbine as follows:

$$
\Delta E_{2}=E_{2, \text { right }}-E_{2, \text { left }}-E_{2, \text { correction }} .
$$

To determine the shaft power produced by the turbine, it is coupled to a generator with an electric load. By measuring the root-mean-square (RMS) voltage, $V_{r m s}$, over one of the three resistors, the total electric power is given by $P_{e}=3 * V_{r m s}^{2} / R$. Since this work is concerned with purely the turbine performance, the influence of the generator is excluded by dividing the electric power with the generator efficiency. This yields the turbine shaft power as follows:

$$
P_{m}=\frac{P_{e}}{\eta_{g e n}},
$$

where the generator efficiency, $\eta_{g e n}$, is calibrated as described in Sec. II C. The RPM of the turbine is also determined from the voltage over the resistor by dividing the measured frequency by the six pole pairs of the generator. The efficiency of the turbine is determined by regarding the shaft power as output and the acoustic power difference as input, i.e.,

$$
\eta_{t}=\frac{P_{m}}{\Delta E_{2}} .
$$

During processing there are automatic checks to exclude any data that are out of the calibrated range or where the generator electric power is not the same as during calibration (probably due to faulty connections). More insight into the exact processing of the experimental data can be found by examining the scripts in the data publication. ${ }^{14}$

\section{Calibration}

The four piezoresistive pressure sensors used in this work are calibrated with respect to a pre-calibrated Kulite XTE-190M pressure sensor. For this purpose, all five sensors are mounted in the same (axial) plane at the left end of the tube section (see Fig. 1). A dynamic calibration is carried out by sweeping the acoustic frequency from 40 to $100 \mathrm{~Hz}$ and the pressure amplitude from 200 to $7500 \mathrm{~Pa}$. Using the reference sensor, all four sensors are calibrated for their sensitivity and their phase difference. The typical sensitivity is $0.5 \mathrm{mV} / \mathrm{Pa}$ with a standard deviation smaller than $0.3 \mu \mathrm{V} / \mathrm{Pa}$ for the entire calibration range. The phase difference is calibrated for each frequency separately, with a maximum mutual difference of $0.1^{\circ}$ over the entire calibration range. More details on the linearity, stability and repeatability of the sensors and its calibration can be found in Appendix B of the work by Oosterhuis. ${ }^{13}$

To calculate the shaft power of the turbine, as given by Eq. (3), the efficiency of the generator must be calibrated for all operating conditions. This is done by coupling the $\mathrm{AC}$ generator to a Maxon A-max 26 DC motor of which the torque constant has been determined to be $13.5 \mathrm{mNm} / \mathrm{A}$. By driving the DC motor and measuring the input current, the torque supplied to the generator is known. The output electric power of the generator is also measured, resulting in the generator efficiency by dividing the electric power with the shaft power (torque times angular velocity). This procedure is carried out in the range of 1000 to $8000 \mathrm{RPM}$ and for resistor sets with $\mathrm{R}=4.7,10$, and $20 \mathrm{Ohm}$. For each of these electrical loads, multiple calibration runs have been carried out, taking care that the RPM range is covered by both ramping up and down and randomly alternating between the two. Note that it is made sure that the rotational direction of the generator is the same as during the experiments and that the voltage is always measured over the same resistor connected to the same coil pair of the generator. As a function of the RPM and the applied load, the measured efficiency of the generator ranges between $40 \%$ and $75 \%$. To show the accuracy and reproducibility of the generator calibration, two different generators have been calibrated and connected to the same turbine setup. The results in Sec. IID show a good agreement in the measured turbine performance for several electrical loads, therewith validating the generator calibration procedure. 


\section{Validation and error estimation}

During the first experiments of this work, the measured performance was varying quite significantly, for example, after remounting a turbine several weeks later. This resulted in some inconsistent results, especially as a function of the acoustic frequency and the generator load. Since the aim of this work is to characterize the turbine performance as a function of these parameters, the experimental procedure needed to be improved. Furthermore, some aspects only have a subtle influence on the turbine performance, such as the effect of the acoustic frequency. Therefore, it needs to be known which measured differences are actually significant and what could be a measurement error. This section will provide the results of the work that has been done to improve and validate the experimental procedure and reduce and quantify the magnitude of several sources of error. This is presented quite extensively in an effort to construct a basis on how bidirectional turbine measurements can be accurately done in thermoacoustics.

Table I provides an overview of the measured turbine efficiency for the validation and error estimation topics treated in this section. To easily compare different measurements, only the maximum efficiency is given for each experiment. The latter was found to be a sufficient measure for comparison since the conclusions do not change when looking at the full acoustic power sweeps. Furthermore, the presented efficiencies are for a single acoustic frequency and resistor set (unless stated otherwise), but it has been confirmed that the results are similar for other operating conditions.

To investigate the repeatability of the measurements, a turbine has been mounted in the experimental setup and kept there for over a month. Table I shows the results of six measurements done during a single day, as well as the efficiency during six days of the month where the largest variations in ambient weather conditions occurred. During the same day, the repeatability of the turbine efficiency is within $0.3 \%$, or within $0.1 \%$ if the unrealistic case of extremely heating the generator and load is neglected. Larger deviations occur during the period of one month, with a maximum difference of around $0.8 \%$ when the atmospheric pressure and temperature are considered constant. In an attempt to have a better repeatability, the ambient pressure and temperature where also logged during the measurements. In the experimental processing, the gas properties of the air have been adjusted accordingly, yielding slightly different values for the calculated acoustic powers [see Eq. (2)]. Including the atmospheric conditions has resulted in a maximum deviation of $0.3 \%$ instead of $0.8 \%$ for the same measurements, showing that the accuracy of the experiments can be significantly improved in this manner.

Another way to improve the measurements is to ensure an accurate phase difference between two adjacent pressure sensors, since even a small phase difference can have a large effect on the calculated acoustic power. The latter becomes clear when varying the pressure sensor locations during a single experiment, which in perfect experiments should not affect the calculated turbine performance. Without incorporating the phase difference measured during calibration, there is a spread of $0.7 \%$ in the efficiency for varying sensor locations (see Table I). This reduces to $0.4 \%$ when including the phase calibration in the acoustic power calculation. Furthermore, there is an average difference of about $0.6 \%$ between the calibrated and non-calibrated case. This shows that for both the absolute value as well as minimizing the

TABLE I. Overview of experimental validation and error estimation results. To easily compare different measurements, only the maximum turbine efficiency, $\eta_{t}$, is given for each experiment. The topics have been investigated with varying turbine designs, so one should only compare the values in each individual row, with the exception of the two rows that treat the influence of atmospheric conditions and phase calibration.

\begin{tabular}{|c|c|c|c|c|c|c|}
\hline & $\eta_{t, 1}[\%]$ & $\eta_{t, 2}[\%]$ & $\eta_{t, 3}[\%]$ & $\eta_{t, 4}[\%]$ & $\eta_{t, 5}[\%]$ & $\eta_{t, 6}[\%]$ \\
\hline Repeatability on the same day & 23.57 & 23.56 & 23.53 & 23.49 & $23.28^{\mathrm{a}}$ & 23.49 \\
\hline Repeatability during one month, with measured atmospheric conditions ${ }^{b}$ & 24.03 & 23.94 & 24.10 & 24.10 & 24.19 & 24.19 \\
\hline Repeatability during one month, with constant atmospheric conditions ${ }^{\mathrm{b}}$ & 23.64 & 23.50 & 23.97 & 24.16 & 24.74 & 23.97 \\
\hline Varying sensor position (with phase calibration) ${ }^{\mathrm{c}}$ & 23.08 & 23.20 & 23.12 & 23.18 & 22.83 & \\
\hline Varying sensor position (without phase calibration) $^{c}$ & 23.80 & 23.91 & 23.73 & 23.85 & 23.25 & \\
\hline Remounting turbine for $4.7 \mathrm{Ohm}(\# 1$ and \#2) and $10 \mathrm{Ohm}(\# 3$ and \#4) & 25.14 & 25.15 & 25.38 & 25.32 & & \\
\hline Varying generator for $10 \mathrm{Ohm}(\# 1$ and \#2) and $20 \mathrm{Ohm}(\# 3$ and \#4) & 23.64 & 23.60 & 23.36 & 23.24 & & \\
\hline Varying tube length with $900 \mathrm{~mm}(\# 1), 1300 \mathrm{~mm}(\# 2)$, and $1700 \mathrm{~mm}(\# 3)$ & 25.75 & 25.51 & 25.21 & & & \\
\hline Reproducibility $3 \mathrm{D}$ prints ${ }^{\mathrm{d}}$ & 24.46 & 24.31 & 24.51 & & & \\
\hline Varying $3 \mathrm{D}$ print technique and material ${ }^{\mathrm{e}}$ & 25.45 & 25.90 & 26.79 & & & \\
\hline
\end{tabular}

${ }^{a}$ This measurement was done with seriously heated resistors and generator by pre-running at maximum power for a considerable amount of time. After cooling down for about half an hour, measurement \#6 was carried out.

${ }^{\mathrm{b}}$ Selected from one month of measurements where the largest variation in atmospheric conditions occurred, with \#1: $1027 \mathrm{hPa}, 18.6^{\circ} \mathrm{C}, \# 2: 1027 \mathrm{hPa}, 18.0^{\circ} \mathrm{C}$, $\#$ 3: $1018 \mathrm{hPa}, 19.3^{\circ} \mathrm{C}$, \#4: $1009 \mathrm{hPa}, 18.9^{\circ} \mathrm{C}$, \#5: $991 \mathrm{hPa}, 19.6^{\circ} \mathrm{C}$, and \#6: $1023 \mathrm{hPa}, 19.5^{\circ} \mathrm{C}$. For the same measurements, results are given where the atmospheric conditions in the processing are set constant at the mean of $1011 \mathrm{hPa}, 18.8^{\circ} \mathrm{C}$.

${ }^{c}$ Each given efficiency is for a different set of pressure sensor locations, while the same turbine is mounted. The sensor locations vary from $50 \mathrm{up}$ to $400 \mathrm{~mm}$ from the edge of the turbine. Results are shown for both neglecting and incorporating the phase calibration of the pressure sensors when calculating the turbine efficiency.

${ }^{\mathrm{d}}$ Results for three 3D printed rotors of the same design and material, manufactured from PA 2200 plastic using Selective Laser Sintering. The values are corrected up to the same tip clearance. Without correction, the efficiencies are $24.38 \%, 24.31 \%$, and $23.82 \%$.

${ }^{\mathrm{e}}$ Results for three 3D printed rotors of the same design but different materials and printing techniques, with \#1: PA 2200 plastic using Selective Laser Sintering, \#2: SR 200 acrylic plastic using material jetting, and \#3: PA 12 nylon plastic using Multi-Jet Fusion. The values are corrected up to the same tip clearance. Without correction, the efficiencies are $25.45 \%, 26.65 \%$, and $27.65 \%$. 
spread for varying sensor locations, including the phase difference is important, even though the calibration only shows a maximum difference of $0.1^{\circ}$ between the sensors (see Sec. IIC). Note that for measurement \#5, one sensor was only $50 \mathrm{~mm}$ from the closed end and $300 \mathrm{~mm}$ from its adjacent sensor, which resulted in a significantly deviating calculated efficiency. Acknowledging a sensor should not be placed there and including the phase calibration, the spread in turbine efficiency is only $0.1 \%$. This independence of the sensor positions gives confidence in the acoustic power calculation and tube correction presented in Sec. II B. As a nuance on this, varying both the total tube length as well as the sensor locations resulted in a larger spread of the efficiency, as shown in Table I. For all results presented in this work, the measurement are done with the intermediate tube length of $1300 \mathrm{~mm}$.

Another possible source of error is the mounting of the turbine in the tube section, since it can be difficult to place the guide vanes straight in the same axial plane of the tube. Especially since their axial length is only $10 \mathrm{~mm}$, the guide vanes tend to twist and rotate when positioning them in the tube. As a consequence, the connected rotor is also not mounted straight, resulting in a changed performance and possibly scraping of the rotor against the outer tube. To prevent this, the guide vanes are mounted by applying force from both sides, and subsequently measuring the axial placement of the guide vane. If there is no significant difference around the whole circumference of both guide vanes, the mount is correct, otherwise it is repeated. By carefully mounting the turbine with this procedure, a maximum difference of $0.1 \%$ in turbine efficiency is measured for different mounts (see Table I), which is a sufficiently small remounting error.

When keeping a single turbine mounted, it is possible to investigate the influence of the generator on the turbine efficiency. In theory, the calibration presented in Sec. IIC should eliminate any influence of the generator by calculating the shaft power based on the calibrated generator efficiency. To validate this procedure, a second generator of the same type has been calibrated and connected to the rotor while keeping the turbine mounted in the tube. Table I shows a maximum difference in turbine efficiency of $0.1 \%$ when comparing both generators for two electrical loads. This demonstrates that the generator calibration is properly carried out and that the turbine performance can be regarded as independent of the used generator.

The final topics presented in Table I are about the accuracy of the 3D printed turbines. This is considered a topic of interest since some dimensions of the turbine design are in the same range as the accuracy of the $3 \mathrm{D}$ printing technique (approximately $0.2 \mathrm{~mm}$ ). Especially the rotor has some small and critical details, e.g., at the tip, which is why three rotor prints of the same design have been ordered to investigate its reproducibility. Two of the rotors have a maximum efficiency within $0.1 \%$. However, the third rotor is found to deviate up to $0.6 \%$. After close examination and measurement of the prints, it was found that the third rotor has about a $0.05 \mathrm{~mm}$ smaller radius. From measurements on the influence of the tip clearance, it was found that a corresponding smaller tip clearance of $0.05 \mathrm{~mm}$ for an unshrouded rotor can account for about $0.7 \%$ in turbine efficiency. Table I presents the efficiencies of the three rotors after they have been corrected according to their relative tip clearance difference. The maximum efficiency spread between the three turbines is now only $0.2 \%$. First of all, this shows the need to measure the actual size of the delivered 3D prints and compare with the designed values. Furthermore, if the efficiency is corrected for the tip clearance effect, the performance is reproducible when re-printing turbines using the same material and technique. The latter is sufficient for this work, since all comparisons will be done using prints from this 3D printing procedure. However, it is interesting to check the turbine performance for different materials and production techniques. Therefore, two additional rotors have been produced from different materials and printing methods. Table I provides production details of these rotors and their performance during measurements. Both of these rotors have a higher maximum efficiency than the rotor used in this work. Even after correcting for the tip clearance, the third rotor yields a $1.3 \%$ higher efficiency. This can be caused by a difference in rotor details due to the printing accuracy (besides a smaller tip clearance), as well as varying material properties. For the latter, especially the surface roughness is important. It is shown that a different surface roughness can have a significant effect on the pressure drop and acoustic losses in Appendix D of the work by Oosterhuis. ${ }^{13}$ On its own, the effect of the surface roughness would already be large enough to explain the measured differences.

From the topics that are treated in this section, it has become clear that calibrating the phase difference of the pressure sensors and accounting for the atmospheric conditions is important to accurately calculate the acoustic power. If a turbine is confirmed to be correctly mounted and the same material and 3D printing technique is used, the experimental procedure is shown to have good reproducibility and a small error. There is not enough data for each topic to calculate proper statistics, but from the presented results, it is reasonable to conclude that any measured difference larger than $0.5 \%$ is not a measurement error but a trustworthy result.

\section{CHARACTERIZATION OF TURBINE PERFORMANCE}

The performance of the bidirectional impulse turbine has been measured for acoustic frequencies of 50, 60, 70, and $80 \mathrm{~Hz}$ with three different electrical loads. For each of these twelve experiments, an acoustic power sweep with approximately fifteen setpoints has been performed. The results from these measurements will be used in this section to characterize the bidirectional turbine performance. This starts with analyzing the velocity diagram in Sec. III A, followed by an overview of the experimental results with several performance indicators in Sec. III B. Subsequently, a dimensional analysis and scaling of the results is done in Sec. III C. Finally, the impedance of the turbine, with a focus on implementing it in a thermoacoustic engine, is presented in Sec. III D. 


\section{A. Velocity diagram}

A conventional way to visualize the flow angles for steady flow turbines is to draw a velocity diagram; see, for example, the turbomachinery book of Dixon. ${ }^{18}$ Such a velocity diagram depicts the magnitude and direction of the flow in the turbine stages. Since the rotor is moving relative to the guidevanes, there is a dinstinction between absolute velocities, c, at angles, $\alpha$, and relative velocities, $\mathrm{w}$, at angles, $\beta$. By assuming the axial velocity is constant and the flow follows the geometry of the blades, the velocity diagram can be drawn. In the simple case of a single guidevane and rotor in steady flow, one can use the velocity diagram to find the most efficient operating point by making sure the relative inlet angle of the rotor, $\beta_{2}$, is equal to the geometric angle of the rotor blade, since there are the least amount of flow losses in this situation. Because the relative velocity and angle depend on two operating variables, namely, the axial velocity, $\mathrm{c}_{x}$, and the blade speed, $\mathrm{U}$, it is useful to define the ratio of these two as the flow coefficient $\phi$,

$$
\phi=\frac{c_{x}}{U} .
$$

Dixon states that, when ignoring Reynolds number effects, the efficiency of the turbine is a unique function of this flow coefficient, with the most efficient operating point as described before. ${ }^{19}$

Now that the relevance of the velocity diagram for steady flow turbomachinery has been made clear, it is interesting to apply this method for the bidirectional impulse turbine in thermoacoustic conditions. Note that the validity of the velocity diagram will be questionable, since there is a quasi-steady axial flow assumed. Besides the effect of the acoustic frequency on the quasi-steady assumption, it is at least not completely valid since the axial velocity on either side of the turbine shows a phase difference of up to a few degrees. However, even in the case the flow is not completely quasi-steady, the analysis could still provide useful details about the flow in the bidirectional turbine.

Figure 4 depicts the velocity diagram at the moment when the axial velocity is at its maximum for a given blade speed. A video of the velocity diagram as the axial velocity changes during the first half of the acoustic period is given in $\mathrm{Mm} .1$ and the data publication. ${ }^{14}$

Mm. 1. Evolution of the velocity diagram during one half of the acoustic period. The velocities and blade speed are taken from the experiment with $70 \mathrm{~Hz}$ acoustic frequency and $10 \mathrm{Ohm}$ generator load at its most efficient setpoint. File of type "mp4" (2.8 MB).

Following the steady flow analysis, for efficient operation the relative inlet angle to the rotor, $\beta_{2}$, should be equal to the $60^{\circ}$ angle of the rotor. Furthermore, the absolute outlet angle of the rotor, $\alpha_{3}$, should equal the $60^{\circ}$ angle of the downstream guidevane to have minimal losses here. The first important thing to notice is that, since the axial velocity is changing during the period while the blade speed is constant, there can only be one axial velocity for which these flow angles are as desired. During the rest of the acoustic period, especially for small axial velocities (see the video), the flow angles are far from optimal. Following this observation it stands to reason that, to get the best flow angles on average, one should focus on the flow angles at the RMS velocity. So, during the first half of the period, one could say that the angle of the upstream guidevane and downstream guidevane should be changed such that the $\beta_{2}$ and $\alpha_{3}$ are optimal at the RMS velocity. However, during the second half of the period the axial flow direction reverses, making the upstream guidevane the downstream guidevane, and vice versa. Changing

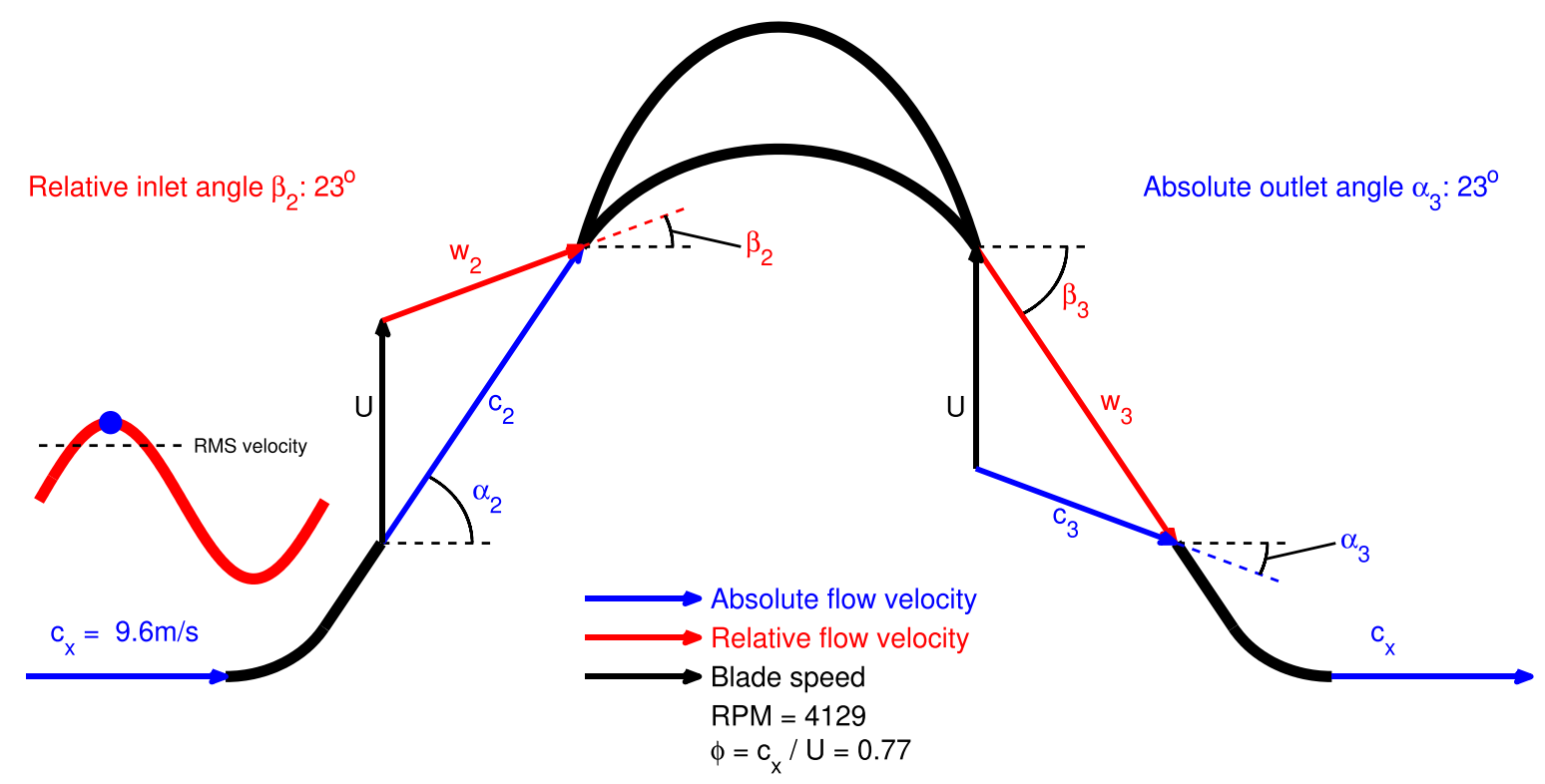

FIG. 4. (Color online) Velocity diagram for the bidirectional impulse turbine at the largest axial velocity during the acoustic period (denoted by the blue dot on the red sinusoidal waveform). The sign convention is such that all angles are taken positive as depicted in this figure. The velocities and blade speed are taken from the experiment with $70 \mathrm{~Hz}$ acoustic frequency and $10 \mathrm{Ohm}$ generator load at its most efficient setpoint. A video of the velocity diagram with varying axial velocity at constant RPM can be found in Mm. 1 and 2 and the data publication (Ref. 14). 
the guidevane angles as mentioned before would provide drastically wrong flow angles during this second half of the period. It is therefore best to stick with the same angle for both guidevanes, as is done in the current work. The consequence of such a design for the flow angles can be seen from Fig. 4. Even for the most efficient setpoint of the turbine and at the maximum axial velocity, the $\beta_{2}$ and $\alpha_{3}$ are only $23^{\circ}$, while ideally they should be $60^{\circ}$. The analysis in this paragraph shows that the main cause for such wrong flow angles is the oscillating nature of the flow, and thus the need for a downstream guidevane with accompanying losses. Therefore, a bidirectional turbine in acoustic flow can never be expected to be as efficient as a conventional turbine in steady flow. Note that in OWCs the flow conditions are sometimes improved by introducing self-pitching guidevanes. ${ }^{20}$ However, these are not considered for thermoacoustic devices since the operating frequency is orders of magnitude higher.

So far, this section has focused on the velocity diagram for the bidirectional turbine at a single setpoint. To investigate the effect of the flow angles on the turbine efficiency, the turbine performance at several setpoints has to be compared. Just as Dixon for steady flow, ${ }^{19}$ the efficiency is examined for varying flow coefficients, now with the RMS values as the corresponding axial velocity. A video showing the velocity diagram, flow coefficient, and turbine efficiency for varying setpoints is given in Mm. 2 and the data publication. ${ }^{14}$

Mm. 2. Velocity diagrams for all setpoints from the experiment with $70 \mathrm{~Hz}$ acoustic frequency and $10 \mathrm{Ohm}$ generator load. File of type "mp4" (1.1 MB).

From the video it can be seen that flow coefficient where the turbine has the best efficiency is at $\phi=0.54$, which is in the same range as reported for this bidirectional turbine design in OWCs. ${ }^{6}$ The flow diagrams show that this most efficient point does not correspond to the best flow angles, as described earlier in this section. The largest turbine efficiency is found where at the RMS velocity the $\beta_{2}$ and $\alpha_{3}$ are nearly horizontal, i.e., $0^{\circ}$, which would be the case for $\phi=0.58$. For the eleven other experiments at different operating conditions, the same holds approximately true, with the flow coefficient at maximum efficiency ranging from 0.49 to 0.63 . Since there is such a spread in this optimum flow coefficient, as well as the fact that the most efficient setpoint is not at the best flow angles, it can be said that the best operating point for the bidirectional turbine in thermoacoustic conditions cannot be concluded from a velocity diagram alone. This can in part be true because the flow cannot be assumed as quasi-steady. However, and more importantly, in the following it will be shown that the maximum turbine efficiency in thermoacoustic conditions is determined by more than just the flow coefficient (and thus velocities) alone.

\section{B. Performance indicators}

In contrast to the thermoacoustic results presented in Sec. III A, similar bidirectional turbines do show a unique dependence of the flow coefficient on the turbine performance for both steady and oscillating flow measurements in OWCs. ${ }^{6,15,21}$ This raises the questions of what is different under thermoacoustic conditions and what additional performance indicators should be considered. To answer the former question, the turbine RPM is shown as a function of the pressure drop amplitude over the turbine, $\Delta P_{a}$, in Fig. 5. First of all, there is a difference in RPM for the varying generator loads (depicted by the different colors). This could be expected since for the same power input one generally sets the turbine RPM by changing its load. However, there is also a clear dependence on the acoustic frequency shown by the different markers in Fig. 5. The same holds true when comparing the axial velocity amplitude to the RPM, albeit to a lesser extent. Following Suzuki et al., ${ }^{15}$ in OWCs scaling the pressure drop with the density and velocity squared leads to a single, linear performance curve with respect to the flow coefficient. This is not the case for the current measurements, where each combination of electrical load and acoustic frequency leads to a different, non-linear curve. The reason for this is that, in thermoacoustics there is (nearly) always a phase difference between the pressure and velocity, as denoted by $\phi_{p U}$ in Eq. (1). Even in traveling wave engines, where in theory a traveling wave has zero phase difference, there is always a standing wave component resulting in at least a few degrees of phase difference. Note that in the current work, a closed tube is used, leading to much larger standing wave ratios and thus phase differences.

To resolve the ambiguity of individually using the pressure drop and velocity as performance indicators, there is a need to combine the two and correctly incorporate the phase difference between them. As can be seen from Eq. (1), the local acoustic power is such a measure. Figure 6 presents the same experimental data as before, but now with the RPM as a function of the acoustic power drop over the turbine. It is interesting to see that, when using the acoustic power drop as a performance indicator, there is no influence of the acoustic frequency on the turbine RPM anymore. Furthermore, the

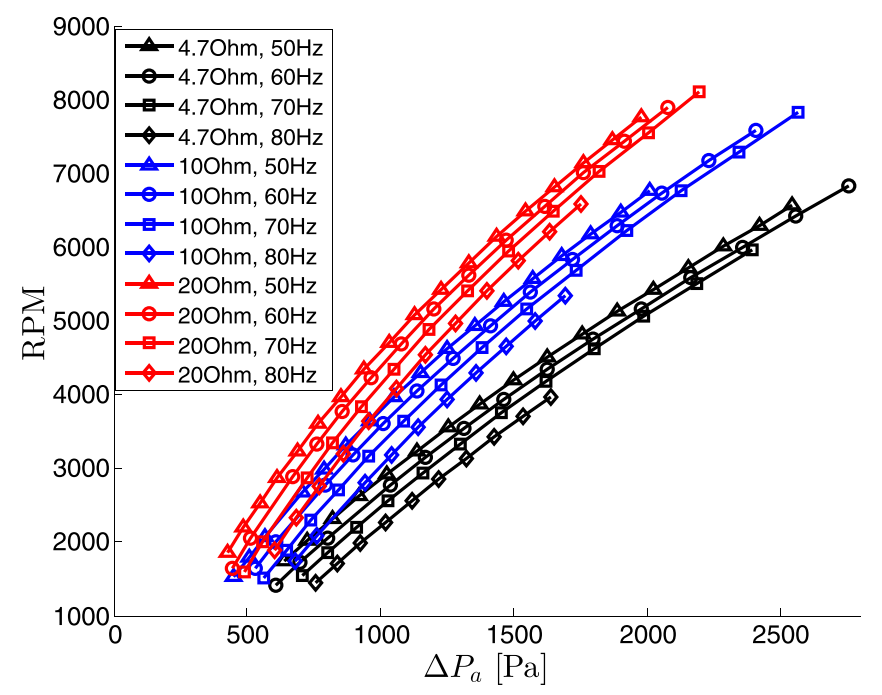

FIG. 5. (Color online) Graph of the measured RPM as a function of the pressure drop amplitude over the turbine. The legend shows the used load resistance and the acoustic frequency for the different measurements. 
curves for all three resistor sets seem to pass through the origin $(0,0)$, which rightfully indicates that there would be no turbine output when there is zero power input. Note that this is not the case when using the pressure drop or velocity amplitude as a performance indicator. The need for combining these two with the correct phase difference shows why the conventional performance characteristics have only limited success in describing the bidirectional turbine in thermoacoustic conditions. Some indicators, such as the turbine efficiency by Setoguchi et $a l .{ }^{6}$ are still valid when correctly incorporating the phase difference. However, to avoid unnecessary errors and provide a clear way to describe the turbine performance, this works continues with using the acoustic power drop as a performance indicator and to calculate the turbine efficiency.

As described in Sec. II B, the turbine efficiency is calculated as the delivered shaft power divided by the acoustic power drop. The efficiency for all operating conditions is given in Fig. 7 as a function of this acoustic power drop. The first thing to notice is that also for the turbine efficiency there is no clear influence of the acoustic frequency. Some small variations might be present as a function of the frequency, but these are not consistent and the differences lie within the measurement error as given in Sec. IID. Therefore, as already suggested by de Blok et al., ${ }^{8}$ the bidirectional impulse turbine performance can be regarded as independent of the acoustic frequency. This is an important result for implementing the turbine in a thermoacoustic engine, since it is not necessary to match the operating frequency of the engine to efficiently run the turbine. This is an advantage over a linear alternator where it can be difficult to match the engine frequency to the resonance frequency of the alternator.

Another interesting thing to note from Fig. 7 is that the maximum efficiency of the turbine is around $25 \%$. This is quite a bit lower than the maximum efficiency of $37 \%$ found for this same turbine in OWCs. ${ }^{6}$ The main difference between the turbine from the OWC and this work is that the current tip clearance of $1 \mathrm{~mm}$ is relatively large. Future work

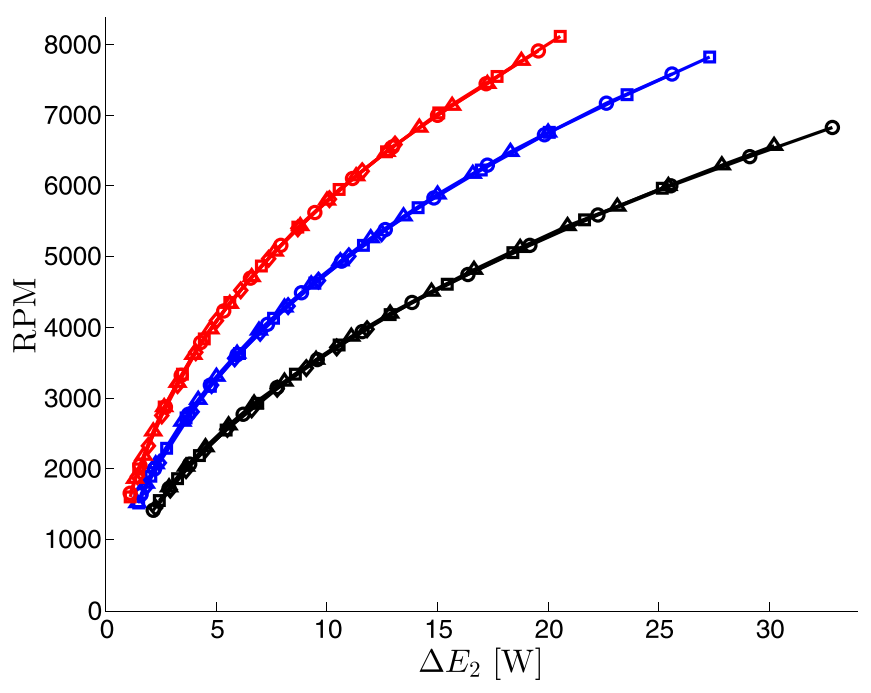

FIG. 6. (Color online) Graph of the measured RPM as a function of the acoustic power drop over the turbine. The legend in Fig. 5 shows the used load resistance and the acoustic frequency for the different measurements.

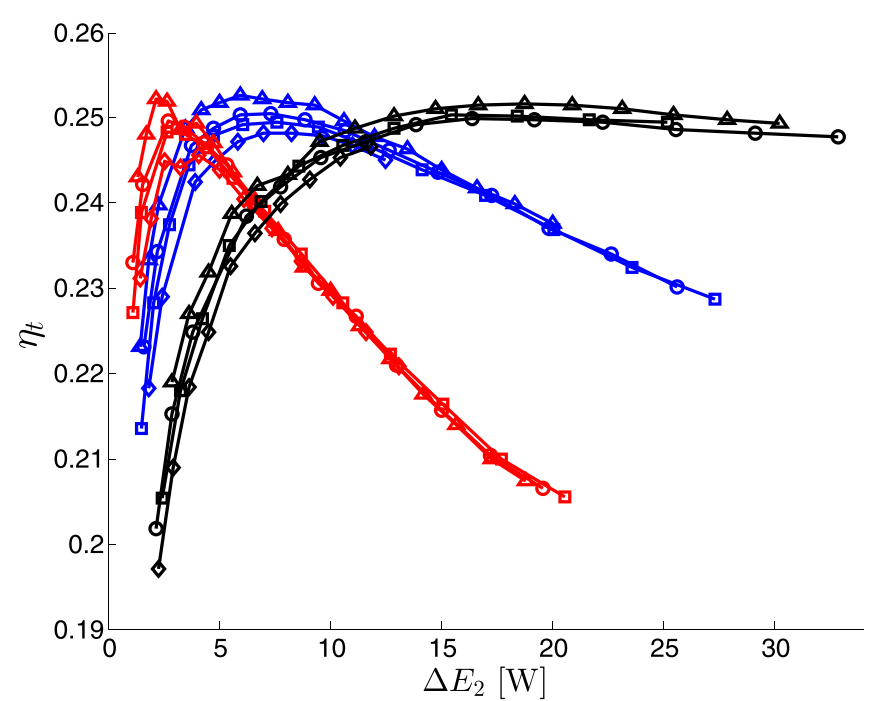

FIG. 7. (Color online) Turbine efficiency as a function of the acoustic power drop over the turbine. The legend in Fig. 5 shows the used load resistance and the acoustic frequency for the different measurements.

should investigate if the same efficiency as in OWCs can be reached with a relatively smaller tip clearance.

The final thing to notice from Fig. 7 is that the efficiency varies significantly as a function of $\Delta E_{2}$ for the different electrical loads, but that the maximum efficiency is the same for all cases. This shows that the turbine can be run at the same maximum efficiency by varying the load for a wide range of operating conditions. To quantify what this load should be in a real engine, Sec. IIIC focuses on finding a performance indicator that scales the turbine efficiency for different loads into a single metric.

\section{Dimensional analysis and scaling}

To find a function that uniquely determines the turbine efficiency, it is first necessary to identify all variables that influence it. For this purpose, a dimensional analysis for geometrically similar machines is performed which assumes the following relationship among the variables:

$$
\eta=f_{1}\left(N, D, \rho, \mu, \Delta E_{2}, c_{x}\right),
$$

with $\mathrm{N}$ the rotation rate, $\mathrm{D}$ the turbine diameter, $\rho$ the density, and $\mu$ the dynamic viscosity. Note that the shaft power is also a dependent function of these same variables, but only the efficiency is considered here. By choosing N, D, and $\rho$ as the repeating variables, the efficiency is found to be a function of the following three dimensionless groups:

$$
\eta=f_{2}\left(\frac{\rho N D^{2}}{\mu}, \frac{c_{x}}{N D}, \frac{\Delta E_{2}}{\rho N^{3} D^{5}}\right)=f_{2}\left(\operatorname{Re}, \phi, \Delta E_{2}^{*}\right) .
$$

By interpolation between the experimental results while keeping $\phi$ and $\Delta E_{2}^{*}$ constant, it is found that the influence of the Reynolds number on the turbine efficiency can be neglected. This is in accordance with results found for classical turbomachinery ${ }^{19}$ and $\mathrm{OWCs}^{22}{ }^{22}$ where for the latter the critical Reynolds is found to be 40000 for the same turbine design and a hub-to-tip ratio of 0.7 . Note that the typical 
Reynolds numbers in this work are in the range of 50000 to 100000 .

What remains is the relationship $\eta=f_{2}\left(\phi, \Delta E_{2}^{*}\right)$, with a yet undetermined function $f_{2}$. By examining the experimental data for all three resistor sets at $70 \mathrm{~Hz}$, it is attempted to find this function. To quantify the success of a proposed function, the points at which the maximum turbine efficiency is achieved are compared. For example, in Sec. III A it was shown that the flow coefficient at maximum efficiency varies for different experiments, which for $70 \mathrm{~Hz}$ is between $\phi=0.54$ for $20 \mathrm{Ohm}$ and $\phi=0.63$ for $4.7 \mathrm{Ohm}$. This equates to a relative difference of $17 \%$, from which it was concluded that the flow coefficient does not uniquely determine the turbine efficiency. Similarly, the influence of the dimensionless acoustic power drop, $\Delta E_{2}^{*}$, is examined. It can be seen from Fig. 8(a) that $\Delta E_{2}^{*}$ is also not a single expression to determine the turbine efficiency with. Especially for large values, which are at small RPMs, there is a big spread between the curves. The maximum efficiency peaks for the different resistor sets also have a relative difference of $45 \%$.

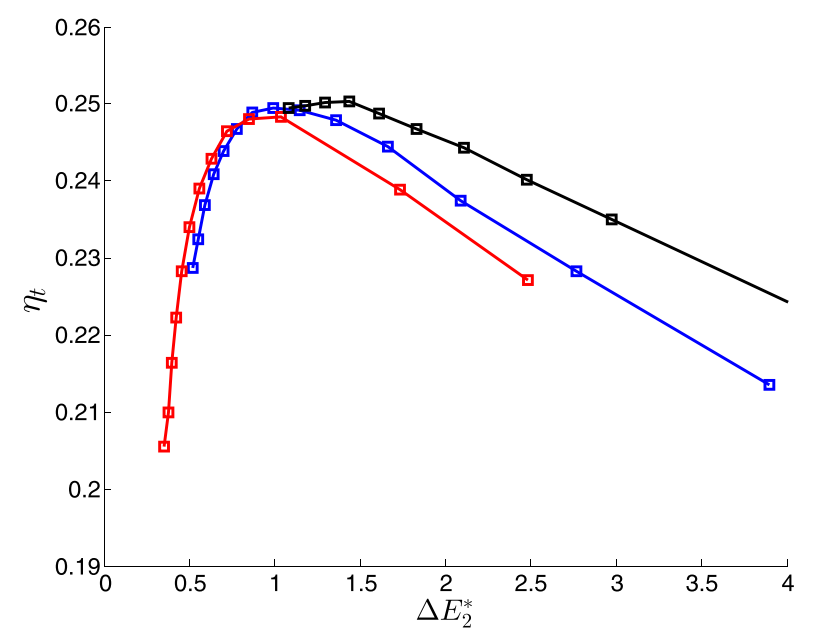

(a)

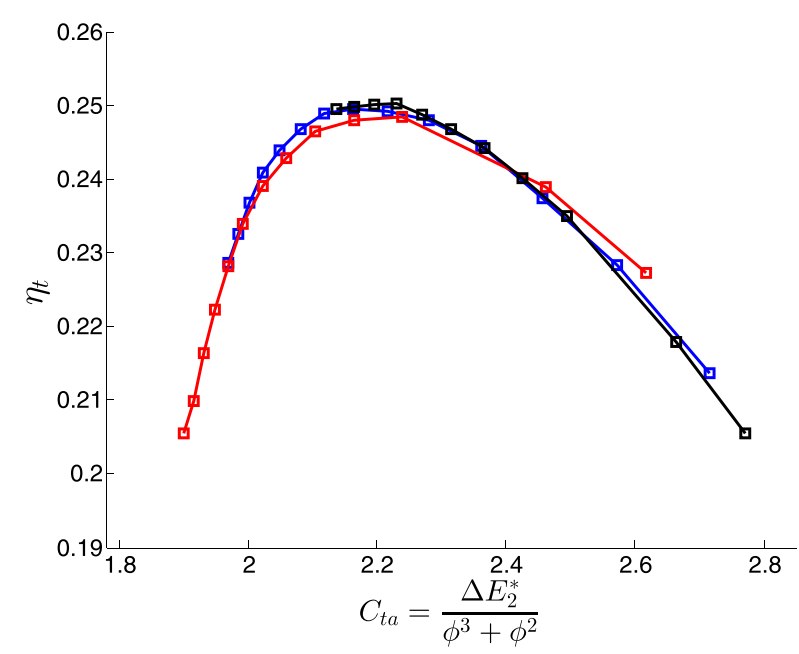

(b)

FIG. 8. (Color online) Turbine efficiency as a function of (a) the dimensionless acoustic power difference and (b) the thermoacoustic input coefficient. The results are for an acoustic frequency of $70 \mathrm{~Hz}$ with the colors for the different resistors sets as show in the legend of Fig. 5 .
From the results presented in the previous paragraph, it is clear that successfully scaling the turbine efficiency has to be done with a function that incorporates both $\phi$ and $\Delta E_{2}^{*}$. A potential function is provided by Setoguchi et al., ${ }^{6}$ who show a scaling for pressure drop times flowrate $(\triangle P Q)$, which they denote by the input coefficient, $C_{A}$. This scaling uses both the axial velocity and the blade speed, $U$, and when applied to $\Delta E_{2}$ is given as follows:

$$
C_{A}=\frac{\Delta E_{2}}{\rho D^{2} c_{x}\left(c_{x}^{2}+U^{2}\right)}=\frac{\Delta E_{2}^{*}}{\phi^{3}+\phi} .
$$

Note that by using $U=N D$, it is possible to rewrite the scaling in terms of $\phi$ and $\Delta E_{2}^{*}$ only, as given on the right hand side of the equation. When examining this scaling with respect to the turbine efficiency, it is found that the maximum efficiency peaks still have a relative difference of $17 \%$. This shows that this is also not a function that uniquely determines the turbine efficiency. However, especially for large and small RPM, this scaling shows more promise than using $\phi$ or $\Delta E_{2}^{*}$ alone. Therefore, following this direction, a new scaling is found. It is referred to as the thermoacoustic input coefficient, $C_{t a}$, and is given as follows:

$$
C_{t a}=\frac{\Delta E_{2}}{\rho D^{2} c_{x}^{2}\left(c_{x}+U\right)}=\frac{\Delta E_{2}^{*}}{\phi^{3}+\phi^{2}} .
$$

In Fig. 8(b), this thermoacoustic input coefficient is shown with respect to the turbine efficiency. It can be seen that the results for the different experiments fall onto a single curve. The peaks have a relative difference of less than $4 \%$, while there is also a good scaling for the operating conditions on either side of this peak. Since including the experiments performed at other acoustic frequencies does not change these conclusions, it can be stated that $C_{t a}$ seems to be a function that uniquely determines the turbine efficiency.

\section{Acoustic impedance}

The final part of this characterization focuses on the specific acoustic impedance of the bidirectional turbine. This is mainly important for implementing the turbine in a thermoacoustic engine, since the impedance difference caused by the turbine directly influences the acoustic conditions, and therewith the performance of the whole device. The impedance of the turbine is a function of its RPM, where the latter can be set by changing the load such that the turbine operates around its most efficient point (according to $C_{t a}$ ). It is therefore interesting to know how the RPM will, in turn, change the acoustic impedance, since that can be used to design an efficient thermoacoustic engine as a whole.

Locally, the specific acoustic impedance is defined as $\mathrm{z}$ $=P_{a} / u_{a}$, with $P_{a}$ the complex pressure amplitude and $u_{a}$ the complex velocity amplitude, which are determined from the measured pressure signals as described in Sec. IIB. The specific acoustic impedance is calculated on both edges of the turbine, and subsequently subtracted from each other to determine the impedance of the turbine, $\mathrm{z}_{t}$. The values are 
normalized by the characteristic specific acoustic impedance, $\mathrm{z}_{0}=\rho c$, where $c$ denotes the speed of sound.

In Fig. 9, the real part (a) and imaginary part (b) of the normalized specific acoustic impedance are given as a function of the turbine RPM. The real part of the impedance represent the in-phase component of the pressure and velocity, and can be regarded as flow resistance. It can be seen that the real part of the impedance increases when more acoustic power is absorbed, either by using a smaller load resistance at a given RPM, or by operating at a larger RPM for the same load. There is also a small influence of the acoustic frequency on the real part of the impedance. For a larger frequency, there is relatively more pressure needed to accelerate the flow, leading to larger values of the impedance. This effect is more clear on the imaginary part of the impedance, which describes the phase difference between the pressure and velocity. The imaginary part of the impedance is positive for all cases, meaning the turbine is an acoustic inertance, which is expected since the turbine causes a narrowing flow path. There is also a small effect of the turbine RPM on the imaginary part of the impedance, but just

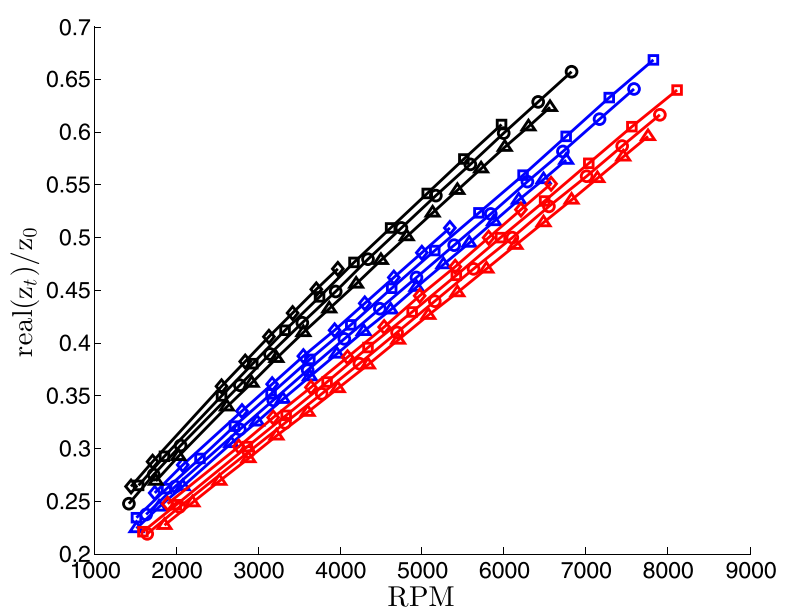

(a)

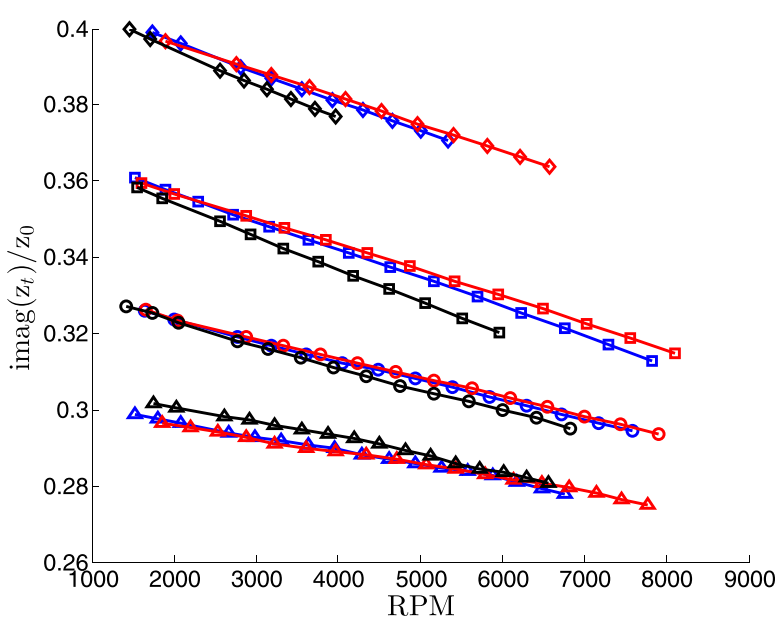

(b)

FIG. 9. (Color online) Real part (a) and imaginary part (b) of the normalized specific acoustic impedance of the turbine as a function of its RPM. The legend in Fig. 5 shows the used load resistance and the acoustic frequency for the different measurements. as for the generator load, this effect is not nearly as large as for the real part of the turbine impedance.

\section{CONCLUSIONS}

The performance of a bidirectional impulse turbine is experimentally characterized for varying thermoacoustic conditions and generator loads. The experimental procedure for these measurements has been validated, during which it is shown that it is essential to incorporate the atmospheric conditions and calibrate the phase of the pressure sensors for accurate measurements. The processing scripts and experimental data from this work can be found in a supplementary data publication. $^{14}$

The maximum turbine efficiency is found to be $25 \%$, which is significantly less than the $37 \%$ found for a similar turbine in an OWC. Future work should focus on increasing the efficiency by optimizing the current design. Reducing the tip clearance and introducing a shroud ring for the rotor are interesting options to achieve this.

From the presented velocity diagram, it is concluded that the flow angles for the bidirectional impulse turbine cannot be optimal due to the sinusoidal velocity and the downstream guidevane. Furthermore, in contrast to conventional turbomachinery and OWCs, the flow coefficient does not uniquely determine the turbine efficiency. It is shown that this is caused by the phase difference between the pressure and velocity, resulting in the need for the acoustic power drop as a performance indicator to combine these two. A dimensional analysis shows that the turbine efficiency is a function of the dimensionless acoustic power drop and the flow coefficient, which are combined into a thermoacoustic input coefficient that does uniquely determine the turbine efficiency.

The specific acoustic impedance of the turbine is presented for varying operating conditions, which can be used to implement the turbine in a thermoacoustic engine. For this purpose, it is found that the acoustic frequency does not influence the efficiency of the turbine itself. This is a big advantage of the bidirectional turbine over a linear alternator, since no frequency matching has to be done to efficiently produce electricity from the acoustic power.

\section{ACKNOWLEDGMENTS}

The authors would like to thank Jos Zeegers and the Eindhoven University of Technology for donating the experimental setup for our thermoacoustic research.

${ }^{1} \mathrm{~K}$. de Blok, "Novel 4-stage traveling wave thermoacoustic power generator," in ASME 2010 3rd Joint U.S.-European Fluids Engineering Summer Meeting collocated with 8th International Conference on Nanochannels, Microchannels, Minichannels, Montreal, Canada (2010), pp. 73-79.

${ }^{2}$ R. Chen and S. L. Garrett, "A large solar/heat-driven thermoacoustic cooler," J. Acoust. Soc. Am. 108(5), 2554-2554 (2000).

${ }^{3}$ Z. Wu, W. Dai, M. Man, and E. Luo, "A solar-powered traveling-wave thermoacoustic electricity generator," Sol. Energy 86(9), 2376-2382 (2012).

${ }^{4}$ D. L. Gardner and C. Q. Howard, "Waste-heat-driven thermoacoustic engine and refrigerator," in Acoustics 2009, Adelaide, Australia (2009), pp. 1-4. 
${ }^{5}$ M. A. G. Timmer, K. De Blok, and T. H. Van Der Meer, "Review on the conversion of thermoacoustic power into electricity," J. Acoust. Soc. Am. 143(2), 841-857 (2018).

${ }^{6}$ T. Setoguchi, S. Santhakumar, H. Maeda, M. Takao, and K. Kaneko, "A review of impulse turbines for wave energy conversion," Renew. Energy 23(2), 261-292 (2001).

${ }^{7}$ A. F. Falcão and J. C. Henriques, "Oscillating-water-column wave energy converters and air turbines: A review," Renew. Energy 85, 1391-1424 (2016).

${ }^{8}$ K. de Blok, P. Owczarek, and M. X. François, "Bi-directional turbines for converting acoustic wave power into electricity," in 9th PAMIR International Conference on Fundamental Applied MHD, Riga, Latvia (2014), pp. 433-438.

${ }^{9}$ E. T. Boessneck and T. E. Salem, "Performance characterization of bidirectional turbines for use in thermoacoustic generator applications," in ASME 2016 10th International Conference on Energy Sustainment, Charlotte, North Carolina (2016).

${ }^{10}$ T. Setoguchi, K. Kaneko, H. Maeda, T. W. Kim, and M. Inoue, "Impulse turbine with self-pitch-controlled guide vanes for wave power conversion: Performance of mono- vane type," Int. J. Offshore Polar Eng. 3(01), 6 (1993).

${ }^{11} \mathrm{C}$. Moisel and T. H. Carolus, "A facility for testing the aerodynamic and acoustic performance of bidirectional air turbines for ocean wave energy conversion," Renew. Energy 86, 1340-1352 (2016).

${ }^{12} \mathrm{P}$. Aben, "High-amplitude thermoacoustic flow interacting with solid boundaries," Ph.D. thesis, Eindhoven University of Technology, 2010.
${ }^{13}$ J. P. Oosterhuis, "Oscillatory flows in jet pumps: Towards design guidelines for thermoacoustic applications," Ph.D. thesis, Universiteit of Twente, 2016.

${ }^{14}$ M. A. G. Timmer, "Characterziation of bidirectional impulse turbines for thermoacoustic engines," Dataset, 4TU Centre for Research Data (2019).

${ }^{15}$ M. Suzuki, M. Takao, E. Satoh, S. Nagata, K. Toyota, and T. Setoguchi, "Performance prediction of OWC type small size wave power device with impulse turbine," J. Fluid Sci. Technol. 3(3), 466-475 (2008).

${ }^{16}$ A. M. Fusco, W. C. Ward, and G. W. Swift, "Two-sensor power measurements in lossy ducts," J. Acoust. Soc. Am. 91(4), 2229-2235 (1992).

${ }^{17}$ G. W. Swift, Thermoacoustics: A Unifying Perspective for Some Engines and Refrigerators, 2nd ed. (ASA Press/Springer, New York, 2017).

${ }^{18}$ S. Dixon and C. Hall, "Axial-flow turbines: Two-dimensional theory," in Fluid Mechanics and Thermodynamics of Turbomachinery, 4th ed. (Butterworth-Heinemann, Oxford, 1998), Chap. 4, pp. 93-133.

${ }^{19}$ S. Dixon and C. Hall, "Dimensional Analysis: Similitude," in Fluid Mechanics and Thermodynamics of Turbomachinery, 4th ed. (Butterworth-Heinemann, Oxford, 1998), Chap. 1, pp. 1-22.

${ }^{20}$ M. Takao, Y. Kinoue, T. Setoguchi, T. Obayashi, and K. A Kaneko, "Impulse turbine with self-pitch-controlled guide vanes for wave power conversion (effect of guide vane geometry on the performance)," Int. J. Rotating Mach. 6(5), 355-362 (2000).

${ }^{21}$ M. Takao and T. Setoguchi, "Air turbines for wave energy conversion," Int. J. Rotating Mach. 2012, 1-10 (2012).

${ }^{22}$ A. Thakker, H. B. Khaleeq, M. Takao, and T. Setoguchi, "Effects of hubto-tip ratio and reynolds number on the performance of impulse turbine for wave energy power plant,” KSME Int. J. 17(11), 1767-1774 (2003). 